\section{COLLIDING DIPOLAR VORTICES IN A STRATIFIED FLUID}

\section{Submitted by G. J. F. van Heijst and J. B. Flor}

\section{(University of Utrecht, The Netherlands)}

The photographs illustrate experiments that have been performed on the collapse of a three-dimensional turbulent patch in a linearly stratified fluid. The turbulence was generated by horizontal injection of a small volume of fluid during a short time interval. A transition to two-dimensional flow occurs when the turbulent patch collapses under gravity, as can be observed from the spectral flux of kinetic energy to larger scales. The collapsed fluid eventually gets organized

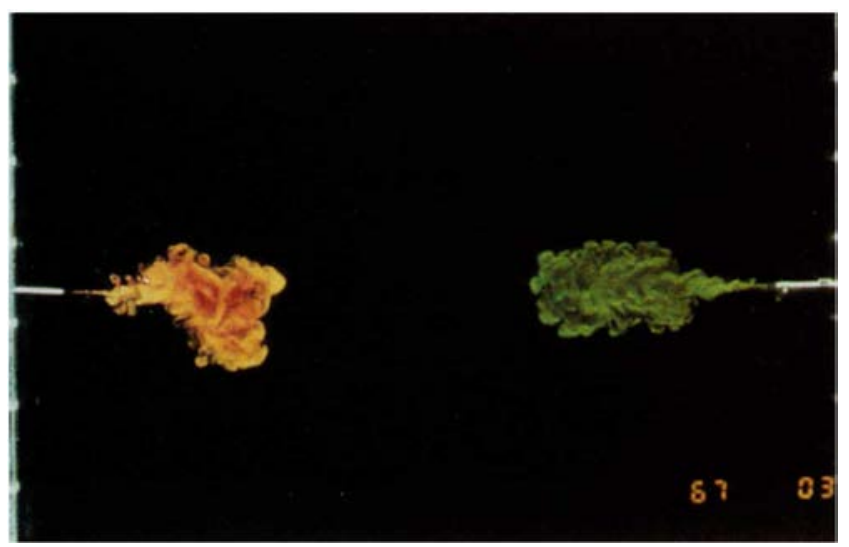

Figure 1(a)

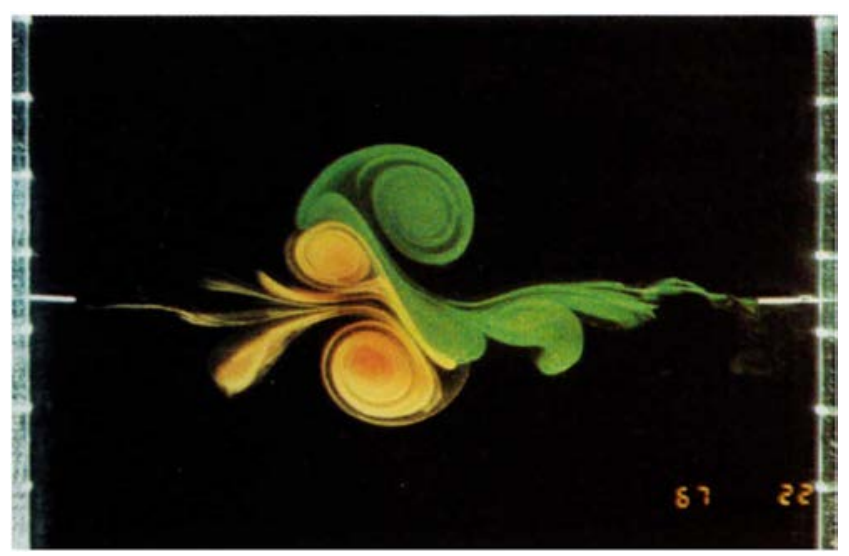

Figure 1(c) in a dipolar flow structure that moves slowly forward along a straight line. The robustness of this dipolar coherent structure is demonstrated in experiments on head-on collisions of two dipoles with approximately identical characteristics. Consecutive stages of a head-on collision are shown by the (plan view) photographs, taken (a) $42 \mathrm{sec}$, (b) $70 \mathrm{sec}$, (c) $120 \mathrm{sec}$, and (d) $225 \mathrm{sec}$ after the injections were stopped. The asymmetry in the observed flow patterns is due to a slight misalignment of the initial dipoles. Nevertheless, the experiment shows nicely that the original dipoles exchange partners, and that two new dipoles emerge, moving along straight lines away from the collision area. Further experimental details are described elsewhere. ${ }^{9}$

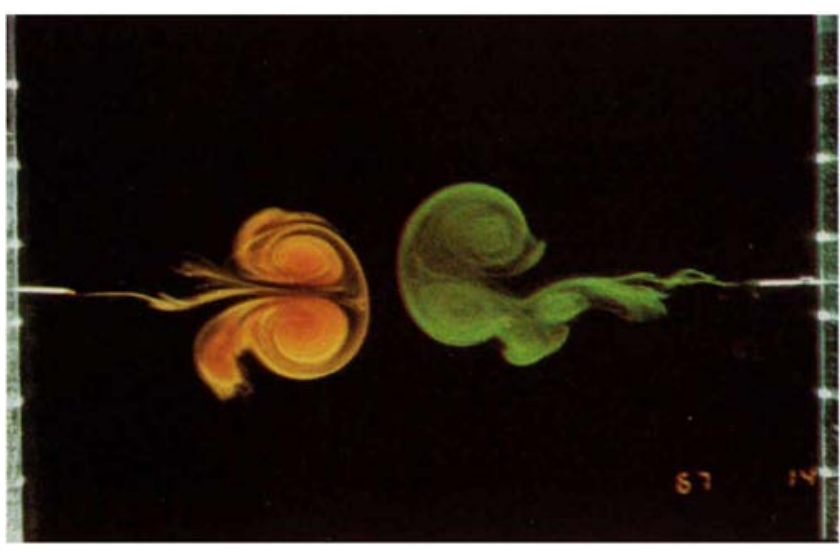

Figure 1(b)

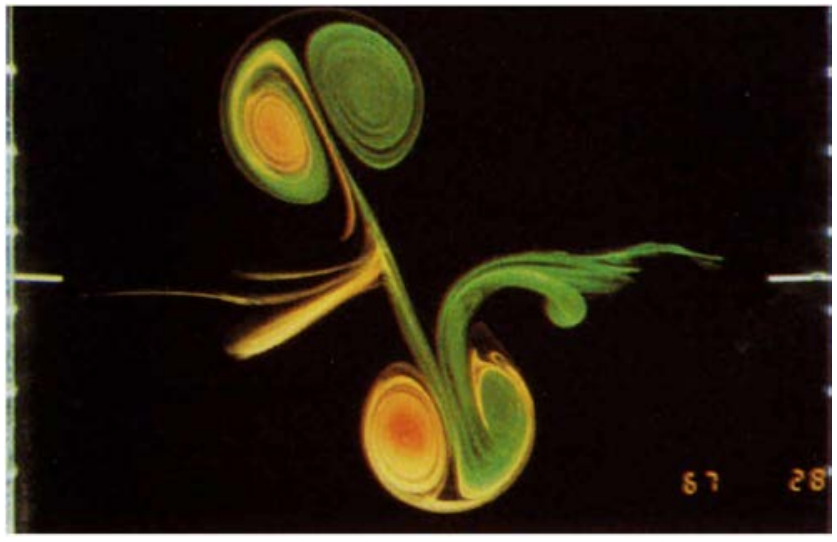

Figure $1(d)$ 